\title{
Effect of geolocation database update algorithms to the use of TV white spaces
}

\author{
Jaakko Ojaniemi ${ }^{1}$, Jussi Poikonen ${ }^{1,2}$, Risto Wichman ${ }^{1}$ \\ ${ }^{1}$ Aalto University, School of Electrical Engineering, Finland \\ ${ }^{2}$ University of Turku, BID Technology, Finland \\ Email: $\{$ jaakko.ojaniemi, jussi.poikonen, risto.wichman\}@aalto.fi
}

\begin{abstract}
According to the European Electronic Communications Committee a practical approach for constructing a cognitive radio network operating in TV white spaces is based on geolocation databases. The practical communication rates achievable for the secondary users and the interference caused to the primary system are fundamentally limited by the information the database has on the geographical distribution of spectrum occupation, and how this information is used to assign transmission power limits to white space devices. In this work we present practical methods for improving the accuracy of the geolocation databases by simulating spectrum sensing samples obtained in a cellular-type white space system operating within the primary TV network area. Furthermore, we analyze the effect of the considered methods on the secondary system throughput and intereference levels on the primary system.
\end{abstract}

Index Terms-cognitive radio, throughput, co-channel interference, database algorithms

\section{INTRODUCTION}

Growth in the numbers of users, devices and services that utilize wireless communication creates pressure to have more spectrum and various types of frequency bands in use. A fundamental objective of cognitive radio (CR) techniques is to enable the use of unutilized and underutilized spectrum resources for wireless communication. Unused spectrum can be taken into use by cognitive radio systems, or cognitive radios can coexist on the reserved frequency bands as secondary users. Cognitive radio systems can offer flexibility in terms of spectrum use and licensing.

In this work, we focus on the use of cognitive radios as secondary devices over primary television broadcast bands, which are the first bands to become available for cognitive radio use. Specifically, we consider white space (WS) systems which take advantage of unused TV frequency bands. As presented for example by the European Electronic Communications Committee (ECC) in [1], according to current knowledge a practical requirement for the implementation of WS systems is the use of a geolocation database which assigns transmission permits to white space devices (WSD) based on their location and the occupation of primary network spectrum. In such scenarios, the practical communication rates achievable for the secondary users and the interference caused to the primary system are fundamentally limited by the information that the controlling database has on the geographical distribution of spectrum occupation, and how this information is used to assign transmission power limits to WSDs. In this work we consider practical methods of improving the accuracy of geolocation databases and their effects on secondary capacity and primary interference levels by building the database from WSD measurements. As the WSDs proliferate, the number of samples in the geolocation database is increasing, and the propagation map can be estimated from the measurements instead of using channel models.

The purpose of the geolocation database in cognitive radio system is to provide information on available frequencies and corresponding maximum allowable emission limits for a WSD operating in a specific location. As the CR network begins its operation, the database calculates the WSD emission limits according to a propagation model of the digital terrestrial television (DTT) network, an arrangement described, e.g., in [2]. However, since these limits are based on predicted DTT electric field strength at a given location and not on real samples, the CR network may not reach its full capacity or on the other hand, DTT reception can experience outage from interfering cognitive radio transmissions. If the WSD is able to sense the DTT field strength in a specific location, this information can be transmitted to the geolocation database which can then recalculate the $\mathrm{CR}$ emission limit.

The DTT field strength is spatially correlated and thereby this correlation can be exploited in approximating the DTT field strength locally. This was addressed also in [3] where statistical techniques for estimating the primary spectrum based on measurement data were presented. Using similar techniques we present a method to build and improve geolocation database content from simulated sensed samples in the spatial domain. As we are interested in the operation of the secondary system we apply these techniques directly to the calculated emission limits and study the effect of different database algorithms to the average uplink throughput and interference levels of the secondary system.

\section{Calculating WSD EMission limits ACCORDing to ECC GUIDELINES}

\section{A. Reference geometry}

As the relative distance between primary receivers and secondary transmitters cannot be known beforehand we must approximate the worst case scenario for the interference caused to the primary transmission. This is done by using a reference geometry to model the propagation path from WSD to DTT receiver, as presented in [1]. It represents the 
worst case scenario where the interference from WSD to DTT receive antenna is the greatest. The coupling gain between the interfering WSD device and the DTT receiver is proportional to carrier frequency, WSD to DTT antenna vertical and horizontal separation and to DTT antenna pattern, gains and losses. For example, according to calculations in [1] based on ITU recommendation BT.419-3 a horizontal separation of $22 \mathrm{~m}$ results in the greatest coupling gain when the vertical separation between transmitting WSD antenna and receiving DTT antenna is $8.5 \mathrm{~m}$. In this calculation a free-space path loss between WSD and DTT was considered. This reference geometry is assumed also when calculating EIRP levels in the following simulations.

\section{B. Approximate method}

In order to calculate the emission limits the database must follow certain criteria. ECC report 159 [1] presents a method for calculating the maximum WSD emission limits that result in interference-free DTT reception after allowed degradation in the location probability. The DTT location probability is defined as the probability with which the mean wanted DTT signal power is greater than the minimum required DTT signal power for acceptable reception. Allowing a certain reduction in location probability, $\Delta q$, the WSD interference can be increased to give the location probability $q_{2}=q_{1}-\Delta q$. Assuming a Gaussian distribution for the variation in DTT signal strength $q_{1}$ is approximated by:

$$
q_{1}=1-\frac{1}{2} \operatorname{erfc}\left(\frac{1}{\sqrt{2}} \frac{m_{S(\mathrm{dBm})}-m_{U(\mathrm{dBm})}}{\sqrt{\sigma_{S(\mathrm{~dB})}^{2}+\sigma_{U(\mathrm{~dB})}^{2}}}\right)
$$

where $m_{S}$ and $m_{U}$ are the DTT signal power and the interfering DTT signal power and $\sigma_{S}$ and $\sigma_{U}$ are their respective standard deviations.

As presented in [1], the approximated WSD emission limits are determined by:

$$
\begin{gathered}
P_{C R(\mathrm{dBm})} \leq m_{D T T(\mathrm{dBm})}-m_{G(\mathrm{~dB})} \\
-r(\Delta f)_{(\mathrm{dB})}-\mu\left(q_{2}\right) \sqrt{\sigma_{D T T(\mathrm{~dB})}^{2}+\sigma_{W S D(\mathrm{~dB})}^{2}}-I M_{d B}
\end{gathered}
$$

where $\mu\left(q_{2}\right)=\sqrt{2} \operatorname{erfc}^{-1}\left(2\left(1-q_{2}\right)\right)$, and $m_{D T T}$ is a median value for the difference between the received DTT signal and the interference by other DTT signals, $m_{G}$ is median coupling gain between WSD and DTT receiver antenna, $r(\Delta f)$ is the protection ratio with a given frequency separation $\Delta f, \sigma_{D T T}$ and $\sigma_{W S D}$ are the standard deviations of the DTT signal power and the WSD to DTT coupling gain, and IM is an additional safety margin. It should be noted that if the coupling gain between the WSD and the DTT receiver is determined using a free-space propagation model, $\sigma_{W S D}^{2}$ becomes zero since the free-space model does not take shadowing or multipath propahation into account, that is, the signal strength at a given distance from the transmitter is fixed.

\section{Simulation AND ANALYSIS METHODS}

\section{A. Simulations}

In simulations, the DTT test network in Turku, Finland is considered. An accurate propagation prediction map based on detailed terrain data of the Turku area is used as a reference. This prediction map was provided by a Finnish DTT broadcast operator and it is considered to represent the true DTT signal power levels in the field. In turn the database calculates the maximum EIRP for the WSD based on its location in the propagation map and updates its own information content with the specified calculation rules. To eliminate the possibility that the inverse complementary error function in (2) would give an undefined value, that is, $q_{2}=q_{1}-\Delta_{q}<0$, the values in the signal map are limited to a minimum DTT signal power of $-103 \mathrm{dBm}$, corresponding to a location probability $q_{1}=0.02$ with the network parameters used. This means that in these locations the allowed degradation in location probabilibity can be selected from interval $(0,0.02)$.

A realistic setup for the geolocation database and sensing based CR system consists of a database containing a propagation prediction of the DTT signal power. This map is adjusted by sensing data from the WSDs. The database is first initialized by the prediction map calculated according to a path loss model with a path-loss exponent of 3.1 and correlated shadowing, and the correlation properties are modeled according to the methods presented in [4]. In short, the shadow fading value follows a log-normal distribution in the linear scale and the two-dimensional spatial correlation of the shadow fading values are obtained through spline interpolation. This initial prediction map is configured according to true site specifications. Fig. 1 shows the both maps, and the goal is to learn the accurate prediction map based on the sensed samples.
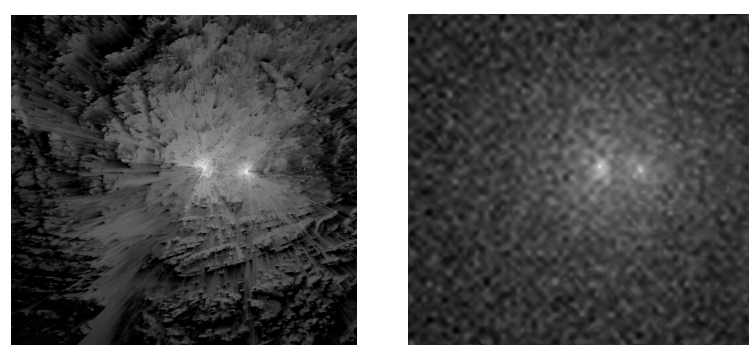

Fig. 1. An accurate propagation prediction map of DTT broadcast signal levels representing true signal levels (left) and a prediction map (right) used in database initialization. The goal is that the database (right) learns from the sensed samples and updates its content so that in turn the secondary emission limits are calculated using accurate estimates of DTT signal levels.

In each turn of the simulation, a number of WSD users, $0.1 \%$ of the population, are moving randomly within the map and sense the DTT signal power in their current pixel. The sensed sample for a single WSD user is simulated as the true DTT signal power given by the accurate prediction map Fig.1 (left) plus an additional random value drawn from a normal distribution with mean $0 \mathrm{~dB}$ and standard deviation $\sigma_{\mathrm{DTT}_{1.5 m}}$ 


\begin{tabular}{l|l}
\hline Parameter & Value \\
\hline \hline$f$ (carrier frequency) $/ B$ (channel bandwidth) & $610 \mathrm{MHz} / 8 \mathrm{MHz}$ \\
\hline$P_{\mathrm{TX} 1} / P_{\mathrm{TX} 2}$ & $1 \mathrm{~kW} / 250 \mathrm{~W}$ \\
\hline$h_{\mathrm{TX} 1} / h_{\mathrm{TX} 2} / h_{\mathrm{WSD}}$ & $40 \mathrm{~m} / 92 \mathrm{~m} / 1.5 \mathrm{~m}$ \\
\hline$\sigma_{\mathrm{WSD}} / \sigma_{\mathrm{DTT}} / \sigma_{\mathrm{DTT}} .5 \mathrm{~m}$ & $5.5 \mathrm{~dB} / 2.5 \mathrm{~dB} / 2.5 \mathrm{~dB}$ \\
\hline$d(\mathrm{shadowing}$ correlation distance) & $550 \mathrm{~m}$ \\
\hline$\Delta q($ allowed decrease in location probability) & $1 \%$ \\
\hline$r(\Delta f)$ co-channel / adj.(N+1) channel & $21 \mathrm{~dB} /-31.9 \mathrm{~dB}$ \\
\hline Number of WSD users & 252 \\
\hline Connection duration (random variable) & {$[0,50]$ turns } \\
\hline$G_{\mathrm{DTT}} / G_{\mathrm{WSD}}$ sense & $9.15 \mathrm{~dB} / 10 \mathrm{~dB}$ \\
\hline pol $/$ ang $($ polarization / angular discrimination) & $3 \mathrm{~dB} / 0.45 \mathrm{~dB}$ \\
\hline WSD sensing reliability & $99.99 \%$ \\
\hline Noise power at CR-BS $N=k T B+N_{f}$ & $-130 \mathrm{~dB}$ \\
\hline
\end{tabular}

TABLE I

SiMULATION PARAMETERS.

$=2.5 \mathrm{~dB}$. This standard deviation was confirmed with field measurements for single-frequency network (SFN) and for pixel sizes less than $100 \mathrm{~m} \times 100 \mathrm{~m}$ [5]. The starting locations and maximum lifetime (connection duration) are assigned randomly for each of the users, although the starting points are limited by the real population density of the considered geographical area in Turku. After the user has disconnected its WSD, user's next starting point, re-connection time and connection duration are again assigned randomly, resulting in a nearly constant number of WSD users simultaneously on the map. Thus, the resulting sampling grid is sparse and irregular. In each turn the sensed sample is uploaded to the database which processes this information according to algorithms presented in section III-B. Simulation parameters are presented Table I.

\section{B. Database algorithms}

In the first stage the database calculates maximum allowed emission power for the WSD in its current pixel. In our simulations it is assumed that the database is aware of the relevant network parameters obtained from the DTT broadcast operator as well as on the device specific protection ratios derived from the WSD's adjacent channel leakage ratio (ACLR) and receiver's adjacent channel selectivity (ACS). In practice this means that it is sufficient that the data uploaded to the database consists of the sensing sample and location of the WSD as we use the same protection ratio for all of the sensing devices.

In simulations, an approximate method is used to calculate the emission limits specified by the uploaded data and network parameters. Since the sensed sample is a function of channel quality it is crucial for the database to use some kind of rules to update its information content so that the information transmitted to the WSD would be as accurate as possible. In the first stage the database simply averages the samples from the same geographical location, that is, the most recent value is the average of the calculated maximum emission limit in (2):

$$
P_{t+1}(m, n)=\frac{\sum_{i=1}^{t} P_{i}(m, n)}{t}
$$

where $P_{i}$ are the previous samples from geolocation $(m, n)$ indicating the propagation map pixel to be updated, and $t$ is the number of corresponding samples. These values are further processed by different database update algorithms which utilize the spatial correlation in DTT signal power. The motivation is that the map representing true DTT signal levels in Fig.1 contains sharp edges and details which should be reproduced. Three algorithms are examined:

(A1) The first algorithm is a 2-dimensional median filter. If there are more than a single WSD user within the geographical area, defined by the window size, the filter selects the median value of the calculated maximum power limit and sets this value in the center pixel:

$$
P_{t+1}(m, n)=\operatorname{median}\left\{P_{s(i, j)}\right\}_{P_{s(i, j)} \neq 0},
$$

where $i=m-\lfloor y / 2\rfloor \ldots m+\lfloor y / 2\rfloor$ and $j=n-\lfloor x / 2\rfloor \ldots n+\lfloor x / 2\rfloor$ and $P_{s(i, j)}$ is a power limit within the filtering window of size $[\mathrm{x}, \mathrm{y}]$. If the number of samples is even the filter takes an average of the two middle values.

(A2) Adaptive Wiener filtering utilizes the local characteristics of an image in the filtering process. A pixel-by-pixel adaptive Wiener filtering can be described by [6]:

$$
\begin{array}{r}
P_{t+1}(m, n)=\frac{\sigma_{P_{s}}^{2}-\sigma_{n}^{2}}{\sigma_{P_{s}}^{2}}\left(P_{s}(m, n)-\mu_{P_{s}}(m, n)\right) \\
+\mu_{P_{s}}(m, n),
\end{array}
$$

where the local mean $\mu_{P_{s}}$ and variance $\sigma_{P_{s}}^{2}$ are the mean and variance of the calculated emission limits within the filtering window, $\sigma_{n}^{2}$ is the mean of all local variances and $P_{s}$ is a matrix containing the calculated maximum WSD emission limits.

(A3) Ordinary kriging is a method regularly used in geostatistics to estimate by interpolation the value of a variable in an unobserved location from observations at known locations. Given a known variogram, a value in a point of a region can be estimated using data in the local neighborhood. This is done by finding the best linear unbiased estimator $\hat{P}[7]$ :

$$
\hat{P}\left(x_{0}\right)=\sum_{i=1}^{n} \omega_{i} P\left(x_{i}\right)
$$

where $\sum_{i=1}^{n} \omega_{n}=1$ are the constrained weights and $P\left(x_{i}\right)$ are the measured samples, in this case the emission limits, in locations $x_{i}$. We use mapping of the locations $x_{i}$ from 2D to 1D for simplifying the notation. The criterion for defining the weights is to minimize the estimation variance with constraint on the weights. The 
estimation variance is:

$$
\begin{array}{r}
\sigma_{E}^{2}\left(x_{0}\right)=E\left[\left(\hat{P}\left(x_{0}\right)-P\left(x_{0}\right)\right)^{2}\right] \\
=-\gamma\left(x_{0}-x_{0}\right)-\sum_{i=1}^{n} \sum_{j=1}^{n} \omega_{i} \omega_{j} \gamma\left(x_{i}-x_{j}\right) \\
+2 \sum_{i=1}^{n} \omega_{i} \gamma\left(x_{i}-x_{0}\right),
\end{array}
$$

where $\gamma(\cdot)$ is a theoretical variogram calculated using a spherical model of the experimental sample semivariogram. Minimizing $\sigma_{E}^{2}$ is an optimization problem whose solution can be found using the Lagrange method. The ordinary kriging system is then obtained as:

$$
\begin{array}{r}
\left(\begin{array}{cccc}
\gamma\left(x_{1}, x_{1}\right) & \cdots & \gamma\left(x_{1}, x_{n}\right) & 1 \\
\vdots & \ddots & \vdots & \vdots \\
\gamma\left(x_{n}, x_{1}\right) & \cdots & \gamma\left(x_{n}, x_{n}\right) & 1 \\
1 & \cdots & 1 & 0
\end{array}\right)\left(\begin{array}{c}
\omega_{1} \\
\vdots \\
\omega_{n} \\
\mu
\end{array}\right) \\
=\left(\begin{array}{c}
\gamma\left(x_{1}, x_{0}\right) \\
\vdots \\
\gamma\left(x_{n}, x_{0}\right) \\
1
\end{array}\right)
\end{array}
$$

where $\mu$ is an additional Lagrange parameter. After solving (8) with respect to $\omega$ the estimate can be obtained using (6).

\section{Throughput calculation}

For analyzing the uplink throughput of the secondary system the network area is divided into hexagonal cells. The purpose of this cellular division is to provide a model for calculating the distances from WSDs to the secondary base stations. This allows the calculation of the received signal power and interference levels at each base station. We use path loss model with path loss exponent 3.1 and shadowing standard deviation $\sigma_{W S D}=5.5 \mathrm{~dB}$ to calculate the received signal power at secondary base stations. The average value for the secondary system throughput can then be evaluated by selecting the combination of transmitter (WSD) and receiver (CR-BS) pairs which maximizes the overall network throughput. QoS criteria and multiple access techniques are not considered and in each cell having active users, only one user is allowed to transmit. It is further assumed that every active WSD transmits at its maximum allowed EIRP over the whole channel bandwidth and that the constructed secondary network is operating at the same frequency band. This is illustrated in Fig. 2. In cell A the user 1 is allowed to transmit whereas in cell B the user which maximizes the total network throughput is selected.

Once the optimal combination of the transmitter and receiver pairs is determined and the corresponding SINR values are calculated for each cell the average cell throughput can be

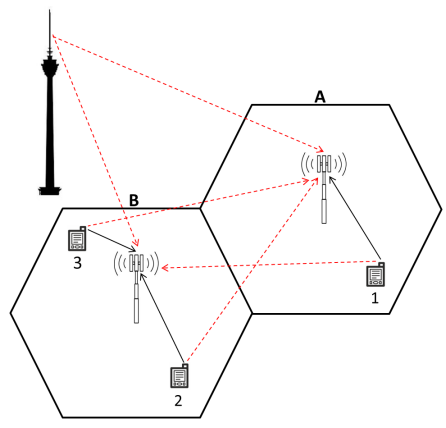

Fig. 2. A co-channel interference scenario representing the possible transmitting WSDs in a cellular network.

calculated with Shannon capacity formula:

$$
\begin{array}{r}
C_{\text {avg }}=\frac{B}{N_{C}} \sum_{i=1}^{N_{C}}\left(1+\frac{P_{i}}{\sum_{j, j \neq i} I_{j}+I_{\mathrm{DTT}_{i}}+P_{N}}\right) \\
=\frac{B}{N_{C}} \sum_{i=1}^{N_{C}}\left(1+\operatorname{SINR}_{i}\right)
\end{array}
$$

where $B$ is the channel bandwidth, $N_{C}$ is the number of used cells, $P_{i}$ is the received power for cell $i, I_{j}$ is the interfering WSD power from cell $\mathrm{j}, I_{D T T_{i}}$ is the interfering co-channel DTT power at cell $i$, and $P_{N}$ is the noise power. As the WSD users are moving randomly within the cells in the simulated network area, the throughput is calculated as an average value over all cells and 2000 simulation turns. Results are presented in section IV-B.

\section{Simulation RESUlts}

\section{A. Evaluation of database algorithms}

Fig. 3 shows the EIRP limits obtained by (2) and algorithms (A0)-(A3). It is notable that using (A0) in (a) the database content corresponds to the reference Fig. 1 only in the city center area where the population density is the highest. Using (A1)-(A3) a larger geographical area can be covered with the same amount of sensing samples. However, filtering may produce inaccurate WSD EIRP limits to some of the pixels, which can result as DTT interference or as CR capacity degradation. The mean square error (MSE) between the true maximum EIRP limits, calculated using the prediction map in Fig. 1 (left), and the database content obtained by using (A0)(A3) is indicated in the figures. MSE1 is the MSE of the pixels where sensing has been performed and $M S E 2$ is the MSE of the pixels which have been processed by (A1)-(A3).

Fig. 4a shows the empirical CDF of the distributions of pixel-wise $P_{\text {max }} / P_{\text {eff }}$ from the pixels which are processed by the presented algorithms after 10,000 iterations. The vertical dashed line represents the optimal staircase function when the database content is the same as the maximum value, calculated from the reference propagation map, in every processed pixel. For example, the left-hand side of the curve represents pixels where the current database content is above the maximum allowed, indicating that the secondary transmission could be 


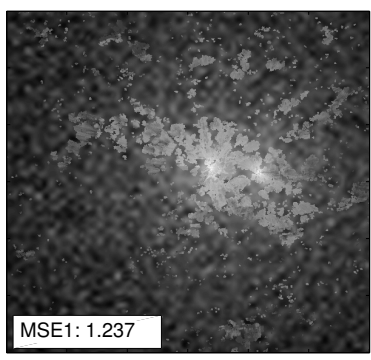

(a)

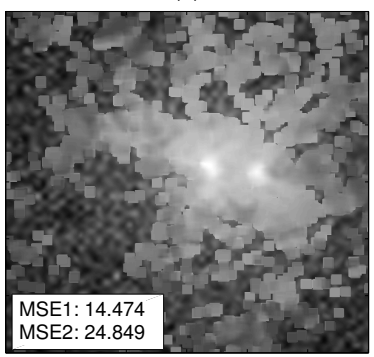

(c)

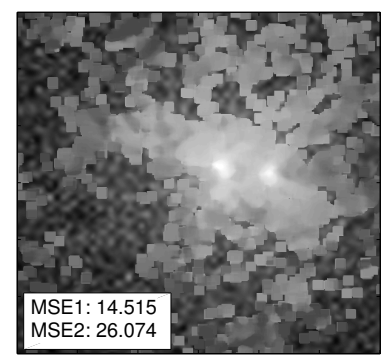

(b)

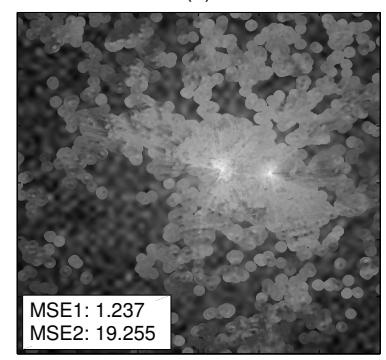

(d)
Fig. 3. Database content after 10,000 iterations using the algorithms (A0)(A3) with the filtering window size of $15 \times 15$ pixels. In (a), only pixels where the sensing samples are available are updated using (A0). In (b)-(d) the sampling data is utilized to span a larger geographical area using the algorithms (A1)-(A3). Note that MSE1 is the same in (a) and (d) which is due to the fact that (A3) in (d) is an exact interpolator which preserves the original samples.

causing interference to the nearby DTT reception. In Fig. 4b, the pixel-wise distribution of $P_{\max } / P_{\text {eff }}$ is taken from the pixels where exact sensing information is available, illustrated in Fig.3a. The smoothing effect of (A1) and (A2) is notable when compared to ordinary kriging (A3). (a)

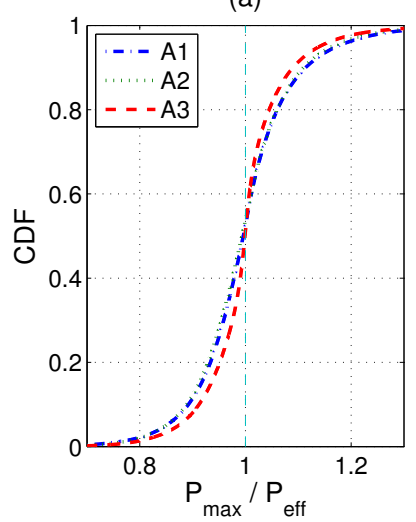

(b)

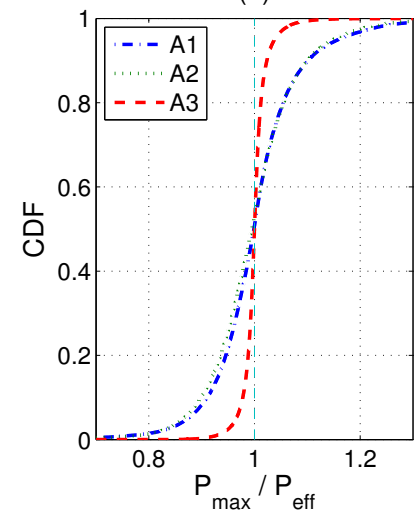

Fig. 4. A distribution of pixel-wise $P_{\max } / P_{\text {eff }}$ of the processed pixels (a) and pixels where the sensing data is available (b) after 10,000 iterations. $P_{\max }$ is the maximum WSD EIRP limit for interference free DTT reception and $P_{\text {eff }}$ is the effective geolocation database value.

\section{B. Network throughput and interference analysis}

For approximating the practical uplink throughput of the secondary system we calculate the mean throughput per cell for co-channel over 2000 iterations using the method presented in III-C for different algorithms. For comparison the adjacent channel $(\mathrm{N}+1)$ average cell throughput is also calculated. Furthermore, we calculate the additional co-channel interference at the primary receiver resulting from the aggregate interference from the transmitting WSDs in other cells. Similarly, for adjacent channel we calculate the average secondary system signal power from other cells at the primary receiver which does not directly affect the DTT reception. In this case the resulting interference from adjacent channel to co-channel is a function of primary receiver ACS and WSD ACLR. Filtering window size of $9 \times 9$ pixels is used, corresponding to the assumed spatial correlation of $500 \mathrm{~m}$ in DTT signal power.

Fig. 5 shows the average cell throughput as a function of cell size. As can be seen the throughput for the co-channel case, less than $0.5 \mathrm{Mbps}$ per cell on average, is negligible. The effect of different database update algorithms on the network throughput is more notable in the adjacent channel case. The reason that using (A3) decreases the average cell throughput is that it maintains most of the allowed EIRP limits near its maximum limit. As all WSDs are transmitting at the maximum allowed power in their current pixel this results in a situation where the aggregate interference from other cells decreases the SINR and therefore the throughput. This can be observed also from Fig. 6 which shows the average received signal power at the primary receiver from transmitting WSDs at other cells, and where the aggregate interference is highest using (A3). In the co-channel case this is interfering power and it can be seen that the achievable SINR is noise limited even with small cell sizes as the received power is below the noise floor. As mentioned, for adjacent channel case the interfering power leakage is a function of primary receiver ACS and WSD ACLR.

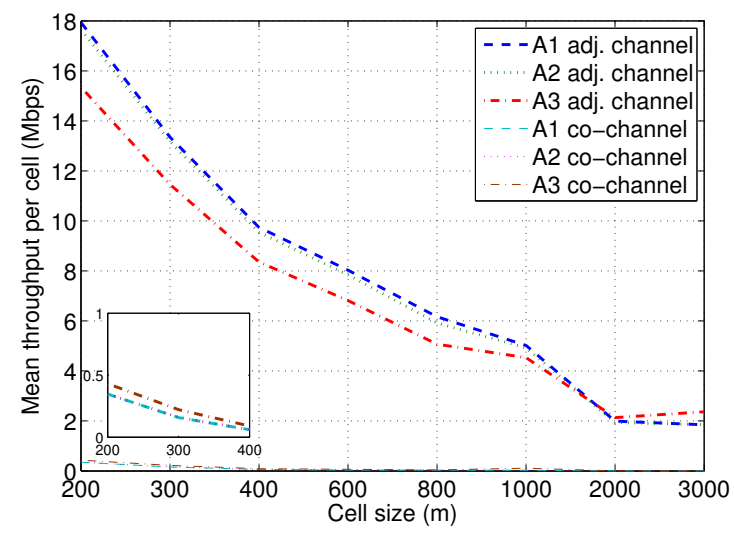

Fig. 5. Average cell throughput as a function of cell size.

Although using (A3) decreases the SINR compared to (A1) and (A2), it should be noted that it is due to the interference limited secondary system, and the performance could be enhanced, for example, with power control techniques. In terms of protecting the primary system transmission algorithm (A3) is most suitable since it preserves the sensing information in a larger number of locations. For example, as indicated in Fig. 


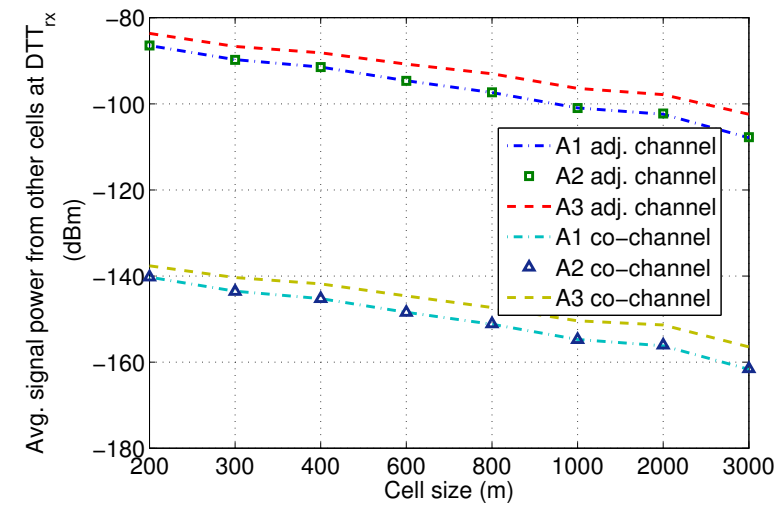

Fig. 6. Average received power levels at the DTT receiver from other cells. Note that the curves do not include the interfering power from the WSD at the same pixel as DTT receiver.

4b, using (A1) or (A2) the WSDs are allowed to transmit above the maximum power limit in about $40 \%$ of the geolocations and thus interfering the primary transmission. The interference probability can be further decreased by using an additional safety margin in calculating the maximum allowed EIRP, as suggested by ECC. However, it will shift the CDF curves in Fig.4 to right-hand side indicating that the power limit is below the maximum allowed in greater amount of pixels, thus there is a trade-off between secondary system throughput and amount of interference caused to the primary system.

\section{CONCLUSION}

We proposed three algorithms for updating sensing and geolocation database driven systems and showed their efficiency with simulations. It was shown that the geographical correlation in DTT signal power can be exploited when processing the uploaded sensing samples, which both improves and speeds the database's information processing determined by the underlying algorithms and their implementation. We also evaluated the achievable throughput in the secondary uplink without multiple access, and showed that in the cochannel case, although the achievable capacity is minimal, it is not affected by the interference from secondary devices from other cells.

\section{ACKNOWLEDGEMENTS}

This work was partly funded by Tekes, the Finnish Funding Agency for Technology and Innovation, in the Trial technology program, and by the Academy of Finland (133888).

\section{REFERENCES}

[1] "Technical and operational requirements for the possible operation of cognitive radio systems in the white spaces of the frequency band 470$790 \mathrm{MHz}$," ECC Report 159, January 2011. Available online through http://www.ero.dk

[2] R. Murty, R. Chandra, T. Moscibroda, P. Bahl, "SenseLess: A databasedriven white spaces network," 2011 IEEE Symposium on New Frontiers in Dynamic Spectrum Access Networks (DySPAN), 3-6 May 2011

[3] J. Riihijarvi, P. Mahonen, M. Wellens, M. Gordziel, "Characterization and modelling of spectrum for dynamic spectrum access with spatial statistics and random fields," IEEE 19th International Symposium on Personal, Indoor and Mobile Radio Communications, 2008. pp.1-6, Sept. 2008
[4] I. Forkel, M. Schinnenburg, M. Ang, "Generation of Two-Dimensional Correlated Shadowing for Mobile Radio Network Simulation," Proceedings of The 7th International Symposium on Wireless Personal Multimedia Communications, WPMC 2004, Sept. 2004

[5] J. Arenas, P. Angueira, I. Pena, U. Gil, L. Fernandez, M. Velez, D. Guerra, "Analysis of the Electric Field Strength Location Variability in the UHF Band for Mobile and Portable Multimedia Broadband Services in SFN Networks," IEEE Broadband Multimedia Systems and Broadcasting (BMSB), 2011.

[6] J. S. Lim, Two-dimensional Signal and Image Processing, Prentice-Hall, New Jersey, 1990. p. 548

[7] H. Wackernagel, Multivariate Geostatistics, Springer, Berlin, 1995. pp. 74-75 